\title{
On Rational Minkowski Pythagorean Hodograph Curves
}

\author{
Jiří Kosinka ${ }^{\mathrm{a}, \mathrm{b}}$, Miroslav Lávička*,b \\ ${ }^{a}$ Centre of Mathematics for Applications, University of Oslo, P.O. Box 1053, Blindern, 0316 Oslo, Norway \\ ${ }^{b}$ University of West Bohemia, Faculty of Applied Sciences, Department of Mathematics, Univerzitni 8, 30100 Plzeň, Czech Republic
}

\begin{abstract}
Minkowski Pythagorean hodograph curves are polynomial curves with polynomial speed, measured with respect to Minkowski norm. Curves of this special class are particularly well suited for representing medial axis transforms of planar domains. In the present paper we generalize this polynomial class to a rational class of curves in Minkowski 3space. We show that any rational Minkowski Pythagorean hodograph curve can be obtained in terms of its associated planar rational Pythagorean hodograph curve and an additional rational function. Moreover, both in the original polynomial and new rational case, we investigate the close relationship between these associated curves in Euclidean plane and Minkowski space.
\end{abstract}

Key words: Pythagorean hodograph curve, medial axis transform, Minkowski space, curvature

\section{Introduction}

Approximation and interpolation algorithms based on planar Pythagorean hodograph (PH) curves provide elegant solutions to many problems that arise when dealing with offsets. PH curves were originally introduced by Farouki and Sakkalis (1990) as a special class of planar polynomial curves. Although a generalization to a rational class followed in Pottmann (1995), the limitations of techniques dealing with rational PH curves still persist. This is due to the fact that for planar rational PH curves only formulas reflecting their dual representations are available. Even though both planar polynomial and rational PH curves have rational offsets, they differ significantly with respect to their arc-length functions. Polynomial PH curves possess polynomial arc-lengths. On the other hand, arc-length functions of rational PH curves may consist of not only rational but also transcendental terms, see Farouki (2008) and the references cited therein.

Even though both planar polynomial and rational Pythagorean hodograph curves admit rational offsets, the usually most demanding part of offset construction is the so called trimming. In practice, not the whole offset but its suitable parts only are used. Details about offset curves trimming can be found e.g. in Elber et al. (1998); Maekawa (1999); Pekerman et al. (2008); Seong et al. (2006). Hence, an alternative approach to the problem based on the medial axis transform of a planar domain plays a crucial role. Using medial axis transform representation makes the trimming procedure of inner offsets very simple - only those parts of the transform where the corresponding circle radius $r$ is less than the offset distance $\delta$ have to be trimmed, cf. Fig. 1. This gives a very strong foundation for studying the so called Minkowski Pythagorean hodograph (MPH) curves introduced in Moon (1999), originally as polynomial curves only. Indeed, if a part of the medial axis transform of a planar domain is a Minkowski Pythagorean hodograph curve, then the corresponding domain boundary segments and all their offsets possess rational parameterizations. This construction naturally associates planar PH curves with spatial MPH curves.

Polynomial PH and MPH curves were thoroughly investigated (see e.g. Choi et al. (2002); Farouki (2002, 2008); Kim and Ahn (2003); Kosinka and Jüttler (2006a); Šír and Kosinka (2010) and the references cited therein) and subsequently used in various algorithms, cf. Farouki et al. (2002, 2004); Jüttler and Mäurer (1999); Kosinka and Jüttler (2006b, 2009); Kosinka and Šír (201x); Š́r and Jüttler (2005, 2007). However, a closed-form characterization

\footnotetext{
* Corresponding author

Email addresses: Jiri.Kosinka@cma.uio.no(Jiří Kosinka), lavicka@kma.zcu. cz (Miroslav Lávička)
}

Preprint submitted to Computer Aided Geometric Design

May 26, 2010 


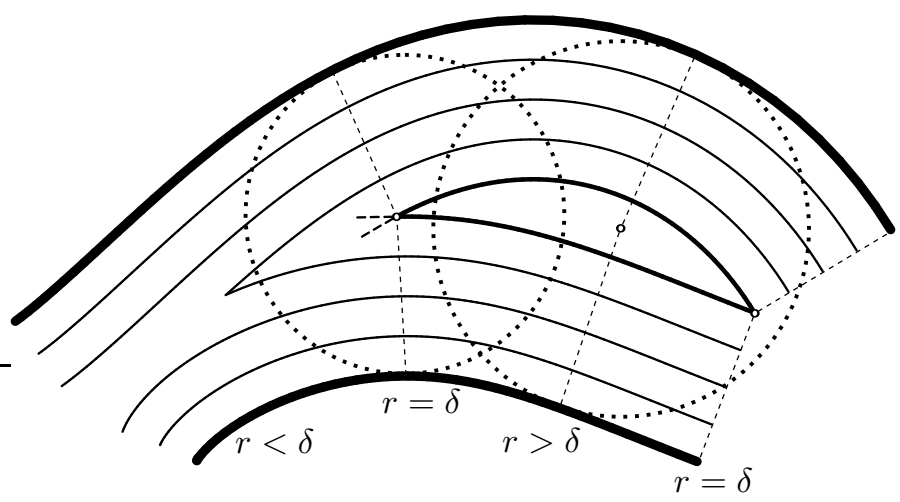

Figure 1: Trimming of inner offsets.

of all spatial rational PH and MPH curves remains an open problem. This is due to two main reasons. First, the dual approach taken in Pottmann (1995) and successfully used on planar rational PH curves is not applicable in space since curves are not hyperplanes in higher dimensions. Second, most of the known characterizations of polynomial PH and MPH curves are based on their hodographs, i.e., first derivative vectors. While this approach works very well in the polynomial case, it cannot be used in a straightforward way in the rational setting. Indeed, starting with a rational hodograph one may arrive at not only rational but also transcendental terms in the parameterization of the curve itself.

The already mentioned close relation between spatial MPH curves and associated planar PH curves motivates us to study the interplay of these special classes of curves in more detail. Pythagorean hodograph algorithms both in Euclidean plane and Minkowski space share many common goals, the main one being rationality of offsets of planar shapes. Since it could be advantageous for better understanding of various approximate/interpolate techniques, it is the purpose of the present paper to address the PH-MPH interconnection thoroughly.

In the next section we recall some basic facts concerning planar PH and spatial MPH curves. Our new contributions are presented in Section 3. Among other results we show that all rational MPH curves in Minkowski 3-space can be obtained in terms of planar rational PH curves and an extra rational function. This approach overcomes the limitations of hodograph representation for MPH curves. We also introduce a modified version of Moon's formula, which turns out to be very advantageous for relating rational and polynomial MPH curves. Moreover, we investigate the close relationship of associated $\mathrm{PH}$ and MPH curves and derive formulas that pertain to their respective curvatures and speeds. As a special case, our results apply to planar rational PH curves and their offsets. Finally, we conclude the paper.

\section{Preliminaries}

In this section we briefly review fundamentals of curves with Pythagorean hodographs in Euclidean plane and Minkowski space.

\subsection{Planar rational curves with rational offsets}

Given a regular $C^{1}$ parametric curve $\mathbf{x}(t)=\left(x_{1}(t), x_{2}(t)\right)^{\top}$, the offset of $\mathbf{x}(t)$ is the set of all points in $\mathbb{R}^{2}$ that lie at a perpendicular distance $\delta$ from $\mathbf{x}(t)$. The two branches of the offset are given by

$$
\gamma_{\delta}(t)=\mathbf{x}(t) \pm \delta \mathbf{n}(t), \quad \mathbf{n}(t)=\frac{\mathbf{x}^{\prime}(t)^{\perp}}{\left\|\mathbf{x}^{\prime}(t)\right\|},
$$

where $\left\|\mathbf{x}^{\prime}(t)\right\|=\sqrt{x_{1}^{\prime}(t)^{2}+x_{2}^{\prime}(t)^{2}}$ and $\mathbf{x}^{\prime}(t)^{\perp}=\left(-x_{2}^{\prime}(t), x_{1}^{\prime}(t)\right)^{\top}$, i.e., $\mathbf{v}^{\perp}$ denotes the rotation of $\mathbf{v} \in \mathbb{R}^{2}$ about the origin by the angle $\frac{\pi}{2}$.

Offset curves are used mainly in numerically controlled machining. They describe the trajectory of a round cutting tool, which is parallel to the cut by a constant distance in the direction normal to the cut at every point. However, even 
for rational $\mathbf{x}(t)$ the rationality of its offsets is generally not guaranteed. A study of offset rationality led to the class of planar Pythagorean hodograph $(\mathrm{PH})$ curves. These curves are defined as rational curves $\mathbf{x}(t)=\left(x_{1}(t), x_{2}(t)\right)^{\top}$ fulfilling the distinguishing condition

$$
\mathbf{x}^{\prime}(t) \cdot \mathbf{x}^{\prime}(t)=x_{1}^{\prime}(t)^{2}+x_{2}^{\prime}(t)^{2}=\sigma(t)^{2}
$$

where $\sigma(t)$ is a rational function, i.e., an element of $\mathbb{R}(t)$, and ' $'$ ' is the standard Euclidean inner product. Since the rationality of a $\delta$-offset curve $\gamma_{\delta}(t)$ of a rational curve only depends on the rationality of the unit normal field $\mathbf{n}(t)$, cf. (1), planar PH curves posses (piece-wise) rational offsets.

Pythagorean hodograph curves were originally introduced by Farouki and Sakkalis (1990) as planar polynomial curves. It was proved (Farouki and Sakkalis, 1990; Kubota, 1972) that the coordinates of hodographs of polynomial PH curves and $\sigma(t)$ form the following Pythagorean triples

$$
\begin{aligned}
x_{1}^{\prime}(t) & =w(t)\left(u^{2}(t)-v^{2}(t)\right), \\
x_{2}^{\prime}(t) & =2 w(t) u(t) v(t), \\
\sigma(t) & =w(t)\left(u^{2}(t)+v^{2}(t)\right),
\end{aligned}
$$

where $u(t), v(t), w(t) \in \mathbb{R}[t]$ are any non-zero polynomials and $u(t), v(t)$ are relatively prime. Hence, the simplest non-trivial example of a planar polynomial PH curve is the so called Tschirnhausen cubic with parameterization $\mathbf{x}(t)=\left(t^{3} / 3-t, t^{2}\right)^{\top}$ obtained for $u(t)=t, v(t)=1, w(t)=1$.

Remark 1. In order to avoid working with piece-wise representations, we consider only curves for which $\sigma(t)>0$ in the interval of interest for the remainder of the paper. Then, $\sigma(t)$ will be called speed of $\mathbf{x}(t)$. This is merely a technical assumption not affecting generality. In cases when $\sigma(t)<0$, one can either substitute $-w(t)$ for $w(t)$ in (3) or consider $|\sigma(t)|$ instead of $\sigma(t)$.

A generalization of polynomial PH curves to rational ones was introduced and studied by Pottmann (1995). This approach uses the dual representation of a plane curve considered as an envelope of its tangents

$$
n_{1}(t) x_{1}+n_{2}(t) x_{2}=h(t), \quad n_{1}(t), n_{2}(t), h(t) \in \mathbb{R}(t) .
$$

In order to guarantee the rationality of (1), the unit normal field $\mathbf{n}(t)$ must rationally parameterize the unit circle. Hence, there must exist relatively prime polynomials $k(t), l(t)$ such that

$$
n_{1}(t)=\frac{2 k(t) l(t)}{k^{2}(t)+l^{2}(t)}, \quad n_{2}(t)=\frac{k^{2}(t)-l^{2}(t)}{k^{2}(t)+l^{2}(t)} .
$$

For the sake of lucidity we omit the dependence on parameter $t$ and write simply $\mathbf{x}$ instead of $\mathbf{x}(t), k$ instead of $k(t)$, etc., whenever no confusion is likely to arise. In addition, to simplify further computations we set $g=h\left(k^{2}+l^{2}\right)$, i.e., the dual representation of an arbitrary PH curve in terms of line coordinates is

$$
\left(2 k l: k^{2}-l^{2}:-g\right) \text {. }
$$

Consequently, a parametric representation of all planar rational PH curves is obtained as the envelope of their tangents given by (6) in the form

$$
x_{1}=\frac{2\left(l l^{\prime}-k k^{\prime}\right) g+\left(k^{2}-l^{2}\right) g^{\prime}}{2\left(k^{2}+l^{2}\right)\left(k l^{\prime}-k^{\prime} l\right)}, \quad x_{2}=\frac{\left(k^{\prime} l+k l^{\prime}\right) g-k l g^{\prime}}{\left(k^{2}+l^{2}\right)\left(k l^{\prime}-k^{\prime} l\right)} .
$$

Differentiating (7) we obtain

$$
x_{1}^{\prime}(t)=n_{2}(t) \sigma(t), \quad x_{2}^{\prime}(t)=-n_{1}(t) \sigma(t),
$$

where $\sigma(t)$, cf. (2), is given by

$$
\sigma=\frac{2 g\left(k^{\prime} l^{\prime \prime}-k^{\prime \prime} l^{\prime}\right)+g^{\prime}\left(k^{\prime \prime} l-k l^{\prime \prime}\right)+g^{\prime \prime}\left(k l^{\prime}-k^{\prime} l\right)}{2\left(k l^{\prime}-k^{\prime} l\right)^{2}}+\frac{g\left(k^{2}+l^{\prime 2}\right)-2 g^{\prime}\left(k k^{\prime}+l^{\prime} l\right)}{2\left(k^{2}+l^{2}\right)\left(k l^{\prime}-k^{\prime} l\right)} .
$$

Furthermore, the representation of offsets can be easily obtained by translating the tangents by a distance $\delta$, i.e., it is sufficient to replace $g(t)=h(t)\left(k(t)^{2}+l(t)^{2}\right)$ by $g(t)=(h(t) \pm \delta)\left(k(t)^{2}+l(t)^{2}\right)$ in (7).

We remark that the concept of planar polynomial PH curves was generalized to 3-dimensional Euclidean space by Farouki and Sakkalis (1994). However, this subject falls beyond the scope of the present paper. 


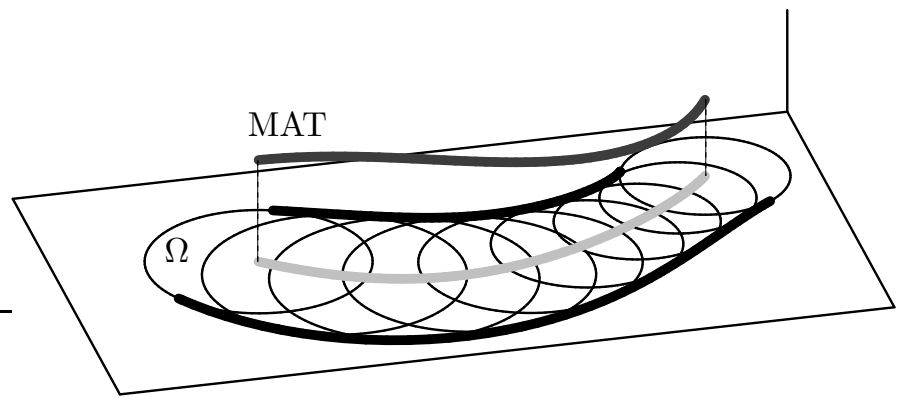

Figure 2: A domain $\Omega$, its maximal inscribed discs, $\operatorname{MA}(\Omega)$ (light grey) and $\operatorname{MAT}(\Omega)$ (dark grey).

\subsection{Medial axis transforms with rational domain boundaries}

Consider a planar domain $\Omega \subset \mathbb{R}^{2}$ and the family of all inscribed discs in $\Omega$ partially ordered with respect to inclusion, see Fig. 2. An inscribed disc is called maximal if it is not contained in any other inscribed disc. Then the medial axis $\operatorname{MA}(\Omega)$ is the locus of all centers $\left(y_{1}, y_{2}\right)^{\top}$ of maximal inscribed discs and the medial axis transform $\operatorname{MAT}(\Omega)$ is obtained by appending the corresponding disc radii $y_{3}$ to the medial axis, i.e., MAT consists of points $\mathbf{y}=\left(y_{1}, y_{2}, y_{3}\right)^{\top}$. We introduce the projection

$$
\mathbb{R}^{2,1} \rightarrow \mathbb{R}^{2}: \quad \mathbf{y}=\left(y_{1}, y_{2}, y_{3}\right)^{\top} \mapsto \stackrel{\nabla}{\mathbf{y}}=\left(y_{1}, y_{2}\right)^{\top},
$$

which naturally relates MAT to MA.

For a given geometric object there is a unique MAT. Conversely, the boundary of an object can be reconstructed from its MAT as the envelope of the one-parameter family of discs. In other words, there is a one-to-one correspondence between the MAT and the domain boundary.

The notion of MAT can be generalized to non-closed shapes. For example for two curve segments (see Fig. 2), we replace maximal discs with discs touching both segments. We will use the notions MA and MAT in this broader sense.

For a $C^{1}$ segment $\mathbf{y}(t)=\left(y_{1}, y_{2}, y_{3}\right)^{\top}$ of $\operatorname{MAT}(\Omega)$ we can compute the corresponding boundary of $\Omega$ using the envelope formula (Choi et al., 1999; Moon, 1999) in the form

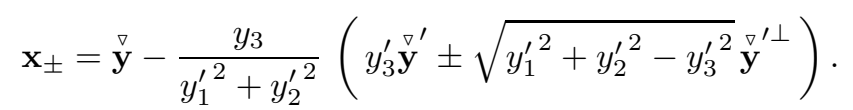

Definition 1. Let $\mathbf{y} \subset \mathbb{R}^{2,1}$ be a curve considered as the MAT of a planar domain and let $\mathbf{x}_{ \pm} \subset \mathbb{R}^{2}$ be given by the envelope formula (11). Then we say that $\mathbf{x}_{+}$and $\mathbf{x}_{-}$are associated with $\mathbf{y}$. Moreover, $\mathbf{x}_{+}$and $\mathbf{x}_{-}$will be called conjugated.

Remark 2. The property of $\mathbf{x}_{ \pm}$being associated with $\mathbf{y}$ can be generalized to the family of translated copies of $\mathbf{y}$ in the $y_{3}$-direction and the corresponding $\delta$-offsets of $\mathbf{x}_{ \pm}$. We note that $\mathbf{x}_{ \pm}$are also known as components of the cyclographic image of $\mathbf{y}$ in the context of Laguerre geometry, see e.g. Peternell and Pottmann (1998); Pottmann and Peternell (1998).

Furthermore, as the points $\mathbf{x}_{+}$and $\mathbf{x}_{-}$are symmetric along the tangent of the medial axis $\stackrel{\nabla}{\mathbf{y}}$ at the point $\left(y_{1}, y_{2}\right)^{\top}$, cf. Fig. 3, we obtain the following relation for conjugated envelope branches $\mathbf{x}_{+}, \mathbf{x}_{-}$associated with $\mathbf{y}$

$$
\mathbf{x}_{-}=\mathbf{x}_{+}-2 \frac{\nabla^{\prime \perp} \cdot\left(\mathbf{x}_{+}-\mathbf{y}\right)}{y_{1}^{\prime 2}+y_{2}^{\prime 2}} \mathbf{y}^{\prime \perp}
$$


A study of rationality of envelopes (11) led to the class of Minkowski Pythagorean hodograph (MPH) curves introduced as polynomial curves by Moon (1999). We define MPH curves as rational curves $\mathbf{y}=\left(y_{1}, y_{2}, y_{3}\right)^{\top}$ in three-dimensional space fulfilling the condition

$$
y_{1}^{\prime 2}+y_{2}^{\prime 2}-y_{3}^{\prime 2}=\varrho^{2},
$$

where $\varrho \in \mathbb{R}(t)$. The PH condition (2) is now fulfilled with respect to the indefinite Minkowski inner product

$$
\langle\mathbf{u}, \mathbf{v}\rangle=u_{1} v_{1}+u_{2} v_{2}-u_{3} v_{3} .
$$

This fact makes the Minkowski space $\mathbb{R}^{2,1}$ the natural ambient space for MPH curves.

The squared norm of a vector $\mathbf{u} \in \mathbb{R}^{2,1}$, defined by $\langle\mathbf{u}, \mathbf{u}\rangle$, can be positive, negative or zero. Hence, we distinguish three types of vectors: a vector $\mathbf{u}$ is called space-like if $\langle\mathbf{u}, \mathbf{u}\rangle>0$, time-like if $\langle\mathbf{u}, \mathbf{u}\rangle<0$, and light-like (or isotropic) if $\langle\mathbf{u}, \mathbf{u}\rangle=0$.

Analogously to the Euclidean case, a necessary and sufficient condition for a spatial polynomial curve to possess a Minkowski Pythagorean hodograph can be expressed in the form

$$
\begin{aligned}
y_{1}^{\prime} & =U^{2}-V^{2}+P^{2}-Q^{2} \\
y_{2}^{\prime} & =2 U V-2 P Q \\
y_{3}^{\prime} & =2 U P-2 V Q, \\
\varrho & =U^{2}+V^{2}-P^{2}-Q^{2}
\end{aligned}
$$

where $U, V, P, Q \in \mathbb{R}[t]$, see Theorem 3.1 of Moon (1999). Again, as in the case of PH curves (cf. Remark 1), we, without loss of generality, restrict ourselves to $\varrho(t)>0$ only. Then, $\varrho(t)$ will be called Minkowski speed.

We emphasize that (15) as well as its planar Euclidean counterpart (3) do not extend in a natural way to rational (M)PH curves. This is due to the fact that we need to integrate the hodograph to obtain the curve itself. Indeed, integrating a general rational function does not yield a rational result.

For later use we recall some basic facts from differential geometry of curves in $\mathbb{R}^{2,1}$. Let $\mathbf{y}(s)=\left(y_{1}, y_{2}, y_{3}\right)^{\top}$ be a sufficiently smooth space-like curve parameterized by arc length and let $\mathbf{T}=\mathbf{y}^{\prime}(s)$ be its unit (space-like) tangent vector, i.e., $\langle\mathbf{T}, \mathbf{T}\rangle=1$. Then the Frenet formulas take the form (i) for space-/time-like $\mathbf{T}^{\prime}$, or (ii) for $\mathbf{T}^{\prime}$ being light-like:

$$
\begin{aligned}
\mathbf{T}^{\prime} & =\varkappa \mathbf{N}, \\
\mathbf{N}^{\prime} & =-\langle\mathbf{N}, \mathbf{N}\rangle \varkappa \mathbf{T}+\tau \mathbf{B}, \quad \text { or } \quad \text { (ii) } \quad \begin{aligned}
\mathbf{T}^{\prime} & =\varkappa \mathbf{N}, \\
\mathbf{N}^{\prime} & =\tau \mathbf{N}, \\
\mathbf{B}^{\prime} & =\tau \mathbf{N},
\end{aligned} \quad \mathbf{B}^{\prime}=-\varkappa \mathbf{T}-\tau \mathbf{B} .
\end{aligned}
$$

The vectors $\mathbf{N}$ and $\mathbf{B}$ are the unit normal and binormal vectors, respectively, and $\varkappa$ and $\tau$ are the Minkowski curvature and torsion of $\mathbf{y}(s)$.

Analogously to Euclidean space, the Minkowski curvature of a (generally non-unit speed) space-like curve $\mathbf{y}$ can be computed using the formula

$$
\varkappa=\frac{\sqrt{\left|\left\langle\mathbf{y}^{\prime} \bowtie \mathbf{y}^{\prime \prime}, \mathbf{y}^{\prime} \bowtie \mathbf{y}^{\prime \prime}\right\rangle\right|}}{\sqrt{\left\langle\mathbf{y}^{\prime}, \mathbf{y}^{\prime}\right\rangle^{3}}},
$$

where $\bowtie$ denotes the cross-product in $\mathbb{R}^{2,1}$ given by

$$
\mathbf{u} \bowtie \mathbf{v}=\left(u_{2} v_{3}-u_{3} v_{2}, u_{3} v_{1}-u_{1} v_{3},-u_{1} v_{2}+u_{2} v_{1}\right)^{\top} .
$$

In addition, let $\kappa_{+}, \kappa_{-}$be the signed curvatures of $\mathbf{x}_{ \pm}$at the contact points with the MAT disc centered at $\stackrel{\nabla}{\mathbf{y}}=\left(y_{1}, y_{2}\right)^{\top}$ with radius $y_{3}$ and $\psi=\angle\left(\mathbf{x}_{+}, \stackrel{\nabla}{\mathbf{y}}, \mathbf{x}_{-}\right)$, see Fig. 3 . Then, as shown in Proposition 2 of Kosinka and Jüttler (2006b), it holds

$$
\left\langle\mathbf{T}^{\prime}, \mathbf{T}^{\prime}\right\rangle=\frac{\kappa_{+} \kappa_{-}}{\left(1+y_{3} \kappa_{+}\right)\left(1-y_{3} \kappa_{-}\right) \sin ^{2} \frac{\psi}{2}}
$$

where $\sin ^{2} \frac{\psi}{2}$ can be computed using the relation

$$
\sin ^{2} \frac{\psi}{2}=1-\cos ^{2} \frac{\psi}{2}=1-\tan ^{2} \phi=\frac{y_{1}^{\prime 2}+y_{2}^{\prime 2}-y_{3}^{\prime 2}}{y_{1}^{\prime 2}+y_{2}^{\prime 2}}
$$




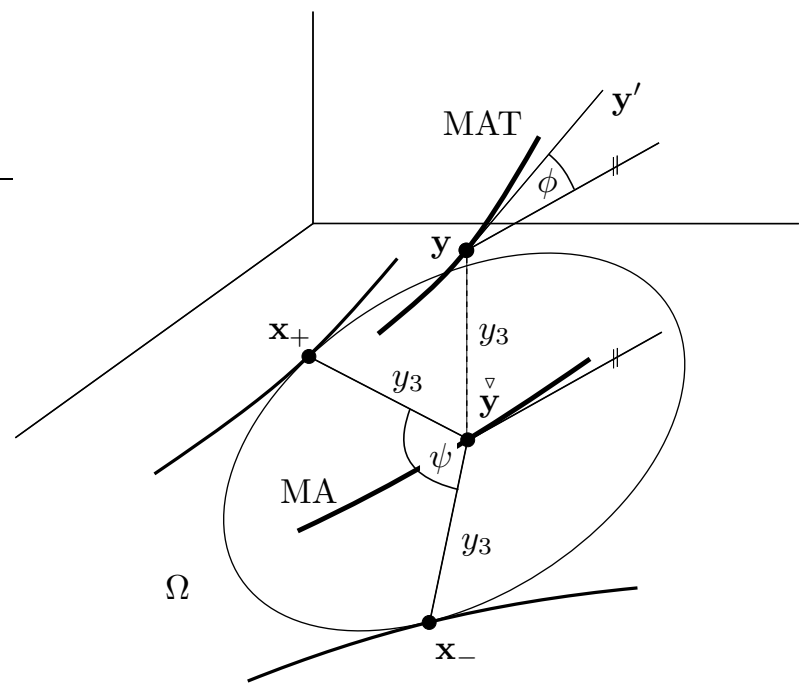

Figure 3: A spatial curve $\mathbf{y}$ considered as $\operatorname{MAT}(\Omega)$, its projection $\stackrel{\nabla}{\mathbf{y}}$ and the associated boundary curves $\mathbf{x}_{+}$and $\mathbf{x}_{-}$.

cf. Section 3.2 of Choi et al. (1999) and Fig. 3. Consequently, if $\left\langle\mathbf{T}^{\prime}, \mathbf{T}^{\prime}\right\rangle \neq 0$, then $\varkappa=\sqrt{\left|\left\langle\mathbf{T}^{\prime}, \mathbf{T}^{\prime}\right\rangle\right|}$ and thus

$$
\varkappa^{4}=\frac{\left(\kappa_{+} \kappa_{-}\right)^{2}}{\left(1+y_{3} \kappa_{+}\right)^{2}\left(1-y_{3} \kappa_{-}\right)^{2} \sin ^{4} \frac{\psi}{2}} .
$$

\section{PH and MPH curves inseparable}

In this section we investigate the close relationship between planar PH curves and spatial MPH curves in both the polynomial and rational case.

\subsection{Polynomial MPH curves}

In order to simplify our further computations, we substitute

$$
a=V-P, \quad b=U-Q, \quad c=V+P, \quad d=U+Q
$$

in (15). Thus we obtain the following

Lemma 1. A polynomial curve $\mathbf{y}=\left(y_{1}, y_{2}, y_{3}\right)^{\top} \subset \mathbb{R}^{2,1}$ is an MPH curve (i.e., $y_{1}^{\prime 2}+y_{2}^{\prime 2}-y_{3}^{\prime 2}=\varrho^{2}$ for some polynomial $\varrho$ ) if and only if there exist four polynomials $a, b, c, d \in \mathbb{R}[t]$ such that the components of the hodograph $\mathbf{y}^{\prime}$ fulfill

$$
\begin{aligned}
y_{1}^{\prime} & =b d-a c \\
y_{2}^{\prime} & =b c+a d \\
y_{3}^{\prime} & =b c-a d \\
\varrho & =b d+a c .
\end{aligned}
$$

Using (23), the envelope formula for polynomial MPH curves simplifies significantly.

Lemma 2. Let y be a polynomial MPH curve given by (23). Then the two envelope curves (11) associated with $\mathbf{y}$ are

$$
\mathbf{x}_{+}=\stackrel{\nabla}{\mathbf{y}}+\frac{y_{3}}{a^{2}+b^{2}}\left(\begin{array}{c}
2 a b \\
a^{2}-b^{2}
\end{array}\right), \quad \mathbf{x}_{-}=\stackrel{\nabla}{\mathbf{y}}-\frac{y_{3}}{c^{2}+d^{2}}\left(\begin{array}{c}
2 c d \\
c^{2}-d^{2}
\end{array}\right) .
$$


In addition, formula (23) yields another interesting result. By computing $\left(\mathbf{x}_{+}^{\prime}\right)^{\perp} / \sigma_{+}$and $\left(\mathbf{x}_{-}^{\prime}\right)^{\perp} / \sigma_{-}$or simply considering $\mathbf{n}_{ \pm}=\left(\stackrel{\nabla}{\mathbf{y}}-\mathbf{x}_{ \pm}\right) / y_{3}$, see Fig. 3, we obtain compact expressions for the unit normal vectors of PH curves $\mathbf{x}_{+}$and $\mathbf{x}_{-}$associated with $\mathbf{y}$ given by (23) in the form

$$
\mathbf{n}_{+}=-\left(\frac{2 a b}{a^{2}+b^{2}}, \frac{a^{2}-b^{2}}{a^{2}+b^{2}}\right)^{\top}, \quad \mathbf{n}_{-}=\left(\frac{2 c d}{c^{2}+d^{2}}, \frac{c^{2}-d^{2}}{c^{2}+d^{2}}\right)^{\top} .
$$

Let us recall the following observation (Choi et al., 1999; Moon, 1999): Let $\Omega$ be a planar domain. If $\operatorname{MAT}(\Omega)$ is a polynomial MPH curve $\mathbf{y}$, then the boundary curves $\mathbf{x}_{ \pm}$of $\Omega$ associated with $\mathbf{y}$ are (piece-wise) rational. Moreover, all offsets of the boundary possess this property as well.

Now we take this observation one step further. In order to obtain $\delta$-offsets it is enough to set $y_{3} \pm \delta$ instead of $y_{3}$ in the envelope formula (24). Hence, we conclude that for any polynomial MPH curve $\mathbf{y} \subset \mathbb{R}^{2,1}$, the curves $\mathbf{x}_{ \pm} \subset \mathbb{R}^{2}$ associated with $\mathbf{y}$ are rational $\mathrm{PH}$ curves. However, since being PH is a property of parameterizations, one may still ask whether the parameterizations in (24) fulfill this property. The answer is given in the following

Lemma 3. The parameterizations (24) associated with a polynomial MPH curve characterized by (23) fulfill the PH condition, i.e., they are rational $P H$ parameterizations.

PROOF. Taking into account (25), one can see that the envelope formula (24) becomes simply

$$
\mathbf{x}_{ \pm}=\stackrel{\nabla}{\mathbf{y}}-y_{3} \mathbf{n}_{ \pm} .
$$

Now, since $\mathbf{n}_{ \pm}$are rational, parameterizations $\mathbf{x}_{ \pm}$satisfy the $\mathrm{PH}$ property.

For the sake of completeness we also provide a direct computation of $\sigma_{ \pm}$. Differentiating $\mathbf{x}_{+}(t)$ with respect to $t$ yields

$$
\sigma_{+}^{2}=\mathbf{x}_{+}^{\prime} \cdot \mathbf{x}_{+}^{\prime}=\frac{\left[\left(a^{2}+b^{2}\right)(a c+b d)+2 y_{3}\left(a^{\prime} b-a b^{\prime}\right)\right]^{2}}{\left(a^{2}+b^{2}\right)^{2}} .
$$

An analogous computation for the conjugated curve $\mathbf{x}_{-}$completes the proof.

We would like to note that the results of this section generalize the theory of polynomial PH curves and their $\delta$-offsets. This can be easily seen by setting $y_{3}(t)=\delta$, or equivalently by imposing $c(t)=w(t) a(t)$ and $d(t)=$ $w(t) b(t)$.

\subsection{Rational MPH curves}

Starting with a spatial polynomial MPH curve $\mathbf{y}$ considered as $\operatorname{MAT}(\Omega)$, we have shown that the boundary of $\Omega$ is a planar PH curve. Moreover, we have proved that the curves $\mathbf{x}_{ \pm}$associated with $\mathbf{y}$ fulfill the PH property.

Now we turn our attention to rational MPH curves. By the same argument as for polynomial MPH curves, it is obvious that spatial rational MPH curves possess planar rational PH curves as their associated curves. Later, we will also show that the associated curves $\mathbf{x}_{ \pm}$are already given in their rational $\mathrm{PH}$ parameterizations.

As a natural question one may ask whether these considerations can be 'reversed'. In other words, given a planar rational $\mathrm{PH}$ curve $\mathbf{x}$, can we construct all rational MPH curves $\mathbf{y}$ such that $\mathbf{x}$ is associated with $\mathbf{y}$ ? In what follows, we show that such a construction is possible. Moreover, we introduce an approach to rational MPH curves overcoming the limitations of hodograph representations.

Definition 2. Let $\mathbf{x}=\left(x_{1}, x_{2}\right)^{\top}$ in $\mathbb{R}^{2}$ be a rational PH curve described by (7) and (9). Let $r$ be an arbitrary rational function. Then we define the curve $\mathbf{y}(\mathbf{x}, r) \subset \mathbb{R}^{2,1}$ as

$$
\mathbf{y}(\mathbf{x}, r)=\left(x_{1}+r n_{1}, x_{2}+r n_{2}, r\right)^{\top},
$$

where $\left(n_{1}, n_{2}\right)^{\top}=\mathbf{x}^{\prime \perp} / \sigma$. 
Remark 3. Turning back to Definition 1 , we see that $\mathbf{x}$ is associated with $\mathbf{y}(\mathbf{x}, r)$. Indeed, $\mathbf{x}$ plays the role of $\mathbf{x}_{-}$. We note that using $\left(n_{1}, n_{2}\right)^{\top}=-\mathbf{x}^{\prime \perp} / \sigma$ in Definition 2 would provide an alternative definition of $\mathbf{y}(\mathbf{x}, r)$, in this case with respect to $\mathbf{x}_{+}$. Moreover, recalling Remark 1 , one can observe that $\sigma<0$ would simply swap the roles of $\mathbf{x}_{+}$ and $\mathbf{x}_{-}$.

Now we present a crucial result concerning rational MPH curves.

Theorem 1. Any rational MPH curve in $\mathbb{R}^{2,1}$ can be expressed in the form of (28).

PROOF. Differentiating $\mathbf{y}(\mathbf{x}, r)$ given by (28) yields

$$
\varrho^{2}=\sigma^{2}(1-r \kappa)^{2}
$$

where $\sigma(t)^{2}=x_{1}^{\prime}(t)^{2}+x_{2}^{\prime}(t)^{2}$ and $\kappa(t)=\left[x_{1}^{\prime}(t) x_{2}^{\prime \prime}(t)-x_{1}^{\prime \prime}(t) x_{2}^{\prime}(t)\right] / \sigma(t)^{3}$ is the curvature of $\mathbf{x}$. Therefore, $\mathbf{y}(\mathbf{x}, r)$ is a rational MPH curve in $\mathbb{R}^{2,1}$.

It remains to show that any rational MPH curve can be expressed in the form (28). To this end we denote $M$ the set of all rational MPH curves in $\mathbb{R}^{2,1}$ and $N$ the set of all parametric curves $\mathbf{y}(\mathbf{x}, r)$ given by (28). Since all $\mathbf{y}$ fulfill the MPH condition, we have that $N \subset M$. On the other hand, let $\mathbf{y}(\mathbf{x}, r) \in M$ be a rational MPH curve. Then the associated boundary curves $\mathbf{x}_{ \pm}$are rational PH curves. By setting $\mathbf{x}=\mathbf{x}_{-}$along with $r=y_{3}$ one can construct $\mathbf{y}(\mathbf{x}, r)$ in the form of elements of $N$. Thus $M \subset N$. This completes the proof.

Summing up, using Theorem 1 and (7), we obtained

Proposition 1. A curve $\mathbf{y} \in \mathbb{R}^{2,1}$ is an MPH curve if and only if there exist two polynomials $k, l$ and two rational functions $g, r$ such that

$$
\left(\begin{array}{l}
y_{1} \\
y_{2} \\
y_{3}
\end{array}\right)=\frac{1}{2\left(k^{2}+l^{2}\right)\left(k l^{\prime}-k^{\prime} l\right)}\left(\begin{array}{c}
2\left(l l^{\prime}-k k^{\prime}\right) g+\left(k^{2}-l^{2}\right) g^{\prime} \\
2\left(k^{\prime} l+k l^{\prime}\right) g-2 k l g^{\prime} \\
0
\end{array}\right)+\frac{r}{k^{2}+l^{2}}\left(\begin{array}{c}
2 k l \\
k^{2}-l^{2} \\
k^{2}+l^{2}
\end{array}\right) .
$$

This characterization can be considered as a rational alternative to Moon's formula (15) or its simplified version (23). For the sake of completeness we also give a formula for $\varrho$ in terms of $k, l, g, r$ :

$$
\begin{aligned}
\varrho^{2}= & \frac{1}{4\left(k l^{\prime}-k^{\prime} l\right)^{4}\left(k^{2}+l^{2}\right)^{2}}\left(g^{\prime \prime}\left(k^{2}+l^{2}\right)\left(k l^{\prime}-k^{\prime} l\right)+g^{\prime}\left[\left(k^{\prime \prime} l-k l^{\prime \prime}\right)\left(k^{2}+l^{2}\right)+2\left(k k^{\prime}+l l^{\prime}\right)\left(k^{\prime} l-k l^{\prime}\right)\right]+\right. \\
& \left.+2 g\left[\left(k^{\prime} l^{\prime \prime}-k^{\prime \prime} l^{\prime}\right)\left(k^{2}+l^{2}\right)+\left(k l^{\prime}-k^{\prime} l\right)\left(k^{\prime 2}+l^{\prime 2}\right)\right]+4 r\left(k l^{\prime}-k^{\prime} l\right)^{3}\right)^{2} .
\end{aligned}
$$

Remark 4. We recall a concept of the so called isotropic surface in $\mathbb{R}^{2,1}$ (Krasauskas and Mäurer, 2000; Peternell and Pottmann, 1998; Pottmann and Peternell, 1998) studied in connection with Laguerre geometry and cyclographic mapping. Starting from a curve $\mathbf{x}(t) \subset \mathbb{R}^{2}$, the corresponding isotropic surface $\Gamma \subset \mathbb{R}^{2,1}$ is described as

$$
\Gamma: \mathbf{y}(t, s)=\stackrel{\Delta}{\mathbf{x}}(t)+s \tilde{\mathbf{n}}(t)
$$

with $\stackrel{\Delta}{\mathbf{x}}=\left(x_{1}, x_{2}, 0\right)^{\top}$ and $\tilde{\mathbf{n}}=\left(n_{1}, n_{2}, 1\right)^{\top}$, where $\mathbf{n}=\left(n_{1}, n_{2}\right)^{\top}$ is the unit normal vector of $\mathbf{x}$. Since it holds

$$
\langle\mathbf{y}(t, s)-\stackrel{\Delta}{\mathbf{x}}, \mathbf{y}(t, s)-\stackrel{\Delta}{\mathbf{x}}\rangle=s^{2}\langle\tilde{\mathbf{n}}, \tilde{\mathbf{n}}\rangle=s^{2}\left(\|\mathbf{n}\|^{2}-1\right)=0,
$$

$\Gamma$ is a ruled surface consisting of straight lines through $\mathbf{x}$ given by light-like vectors $\mathbf{y}(t, s)-\stackrel{\Delta}{\mathbf{x}}(t)$.

Furthermore, if $\mathbf{x}(t)$ is a PH curve then $\Gamma$ is a (piece-wise) rational surface. Hence, we can conclude that all MPH curves $\mathbf{y}(t)$ given by (28) are rational curves on the rational surface (32) obtained by setting $s=r(t) \in \mathbb{R}(t)$. Starting from conjugated $\mathrm{PH}$ curves $\mathbf{x}_{+}(t)$ and $\mathbf{x}_{-}(t)$, the corresponding isotropic surfaces $\Gamma_{+}$and $\Gamma_{-}$intersect in the associated MPH curve $\mathbf{y}(t)$, cf. Fig. 4. Furthermore, planar sections of $\Gamma_{ \pm}$lead to $\delta$-offsets of the associated domain boundaries.

In addition, we would like to note that analogous observations were made for PN and MOS surfaces in $\mathbb{R}^{3,1}$ in Peternell (2010). It was proved that MOS surfaces possess rational isotropic normal vector fields and can be characterized as two-dimensional rational subvarieties of rational isotropic hypersurfaces in $\mathbb{R}^{3,1}$. 


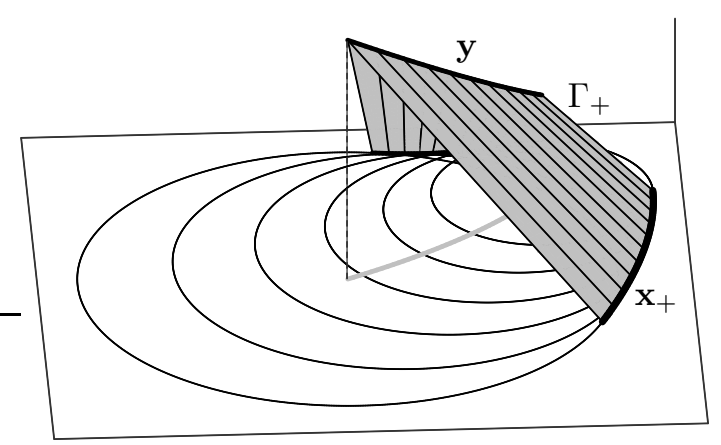

Figure 4: Isotropic surface $\Gamma \subset \mathbb{R}^{2,1}$ corresponding to $\mathbf{x} \subset \mathbb{R}^{2}$ and $\mathbf{y} \subset \mathbb{R}^{2,1}$.

With the help of (28), one can determine the degree of the resulting MPH curve depending on the degree of $\mathbf{x}(t)$ and $r(t)$.

Lemma 4. Let $\mathbf{x}$ be a rational PH curve of degree $d_{1}$ and $r$ be of degree $d_{2}$. Then the degree of the associated $\mathbf{y}$ is at most $5 d_{1}+d_{2}-1$. In the case of polynomial $\mathbf{x}$ and $r$ the degree of $\mathbf{y}$ amounts to at most $\left(d_{1}-1+\max \left(d_{1}, d_{2}\right)\right) /\left(d_{1}-1\right)$.

For an arbitrary rational MPH curve, analogously to Lemma 3, we obtain

Lemma 5. The parameterizations (11) of curves $\mathbf{x}_{ \pm}$associated with a rational MPH curve (28) fulfill the PH condition, i.e., they are rational $P H$ parameterizations.

PROOF. Applying the envelope formula (11) to the MPH curve $\mathbf{y}(\mathbf{x}, r)$ given by (28), we arrive at

$$
\mathbf{x}_{ \pm}=\stackrel{\nabla}{\mathbf{y}}-\frac{r}{\varrho^{2}+r^{\prime 2}}\left(r^{\prime} \stackrel{\nabla}{\mathbf{y}}^{\prime} \pm \varrho \mathbf{\nabla}^{\prime \perp}\right)
$$

Using (29) we see that either $\varrho=\sigma(1-r \kappa)$ or $\varrho=-\sigma(1-r \kappa)$ holds. Since the latter case only swaps $\mathbf{x}_{+}$and $\mathbf{x}_{-}$ and produces insignificant sign changes in the formulas below, we consider for the sake of simplicity the former case only. Then, (34) simplifies to

$$
\begin{aligned}
& \mathbf{x}_{-}=\mathbf{x}, \\
& \mathbf{x}_{+}=\mathbf{x}+\frac{2 r \varrho}{\sigma\left(r^{\prime 2}+\varrho^{2}\right)}\left(\begin{array}{c}
r^{\prime} x_{1}^{\prime}-\varrho x_{2}^{\prime} \\
\varrho x_{1}^{\prime}+r^{\prime} x_{2}^{\prime}
\end{array}\right),
\end{aligned}
$$

where $\sigma(t)^{2}=x_{1}^{\prime}(t)^{2}+x_{2}^{\prime}(t)^{2}$ and $\varrho(t)$ is as in (29), or (31). The result for $\mathbf{x}_{-}$is obvious. Thus, it remains to show that $\mathbf{x}_{+}$also fulfills the $\mathrm{PH}$ condition. Differentiating $\mathbf{x}_{+}(t)$ given by (36) yields

$$
\mathbf{x}_{+}^{\prime} \cdot \mathbf{x}_{+}^{\prime}=\left(\frac{\sigma{r^{\prime}}^{2}+2{r^{\prime}}^{2} \varrho+\sigma \varrho^{2}-2 r \varrho r^{\prime \prime}+2 \varrho^{3}}{r^{\prime 2}-\varrho^{2}}\right)^{2} .
$$

This completes the proof.

Obviously, polynomial MPH curves, given by (15) or (23), must form a proper subset of the set of rational MPH curves described by (30). A natural question arises: How do we choose $g(t)$ and $r(t)$ in Proposition 1 in order to obtain a polynomial MPH curve? It turns out that we can efficiently adapt the approach for relating planar rational and polynomial PH curves used in Farouki and Pottmann (1994), as shows the following 
Theorem 2. Any polynomial MPH curve in $\mathbb{R}^{2,1}$ can be obtained using (30) by setting

$$
\begin{aligned}
g(t) & =2 k l \int(k m-l n) \mathrm{d} t-\left(k^{2}-l^{2}\right) \int(k n+l m) \mathrm{d} t-\left(k^{2}+l^{2}\right) \int(l m-k n) \mathrm{d} t, \\
r(t) & =\int(l m-k n) \mathrm{d} t
\end{aligned}
$$

where $k(t), l(t), m(t), n(t)$ are arbitrary polynomials.

PROOF. For the sake of brevity we do not go into details and refer the reader interested in the derivation of the given expressions to Farouki and Pottmann (1994). Consider $g$ and $r$ in the form (38). Substituting $g$ and $r$ into (30) yields

$$
y_{1}(t)=\int(k m-l n) \mathrm{d} t, \quad y_{2}(t)=-\int(k n+l m) \mathrm{d} t, \quad y_{3}(t)=\int(l m-k n) \mathrm{d} t .
$$

These expressions give a polynomial MPH curve of type (23) for $a=l, b=-k, c=n$ and $d=-m$. The fact that (23) describes all polynomial MPH curves completes the proof.

\subsection{Curvatures of associated curves}

Motivated by a potential $G^{2}$ Hermite interpolation scheme for MPH curves, we focus on some relations between the Minkowski curvature of an MPH curve and the curvatures of its associated planar PH curves. Let us emphasize that the results discussed in the following paragraphs (including formula (29)) hold also for general MATs and the associated curves $\mathbf{x}^{ \pm}$, i.e., without the assumption of rationality of $\varrho, \sigma_{ \pm}, \varkappa$ and $\kappa_{ \pm}$.

Let $\mathbf{x}_{+}$and $\mathbf{x}_{-}$be two conjugated PH curves with curvatures $\kappa_{+}, \kappa_{-}$and speeds $\sigma_{+}, \sigma_{-}$, respectively. Defining $\mathbf{y}\left(\mathbf{x}_{-}, r\right)$ we obtained the relation $\varrho_{-}^{2}=\sigma_{-}{ }^{2}\left(1-r \kappa_{-}\right)^{2}$, cf. (29). On the other hand, using $\mathbf{x}_{+}$, cf. Remark 3, we arrive at $\varrho_{+}^{2}=\sigma_{+}{ }^{2}\left(1+r \kappa_{+}\right)^{2}$. Since $\mathbf{x}_{+}, \mathbf{x}_{-}$are conjugated, the associated MPH curves coincide, i.e., $\mathbf{y}\left(\mathbf{x}_{-}\right)=\mathbf{y}\left(\mathbf{x}_{+}\right)$. Consequently, the Minkowski speeds are equal $\varrho_{-}=\varrho_{+}$and

$$
\sigma_{+}^{2}\left(1+r \kappa_{+}\right)^{2}=\sigma_{-}^{2}\left(1-r \kappa_{-}\right)^{2} .
$$

If we multiply $\varrho_{+}^{2}$ and $\varrho_{-}^{2}$ and compare the result with (21) we arrive at

$$
\left(\sigma_{+} \kappa_{+}\right)^{2}\left(\sigma_{-} \kappa_{-}\right)^{2}=\left(\varrho \varkappa \sin \frac{\psi}{2}\right)^{4}
$$

which holds for MPH curves with space- or time-like vector $\mathbf{T}^{\prime}$. Otherwise, $\varkappa=0$ or $\varkappa=1$, see Section 2.4 of Kosinka and Jüttler (2006b). Using $\sin ^{2} \frac{\psi}{2}=\varrho^{2} /\left(\varrho^{2}+{r^{\prime}}^{2}\right)$, cf. (20), we obtain

$$
\left(\sigma_{+} \kappa_{+}\right)^{2}\left(\sigma_{-} \kappa_{-}\right)^{2}=\left(\frac{\varrho^{4} \varkappa^{2}}{\varrho^{2}+r^{\prime 2}}\right)^{2}
$$

Furthermore, computing $r$ and its derivative using (40) and then substituting into (42), one can derive a relation for only curvatures $\kappa_{+}, \kappa_{-}, \varkappa$ and parametric speeds $\sigma_{+}, \sigma_{-}, \varrho$ of the conjugated PH curves $\mathbf{x}_{+}, \mathbf{x}_{-}$and their associated MPH curve $\mathbf{y}$.

Now we apply our results to the special case when $r \equiv \delta$ is constant. Then $\mathbf{y}=\left(y_{1}, y_{2}, \delta\right)^{\top}$ is a planar PH curve satisfying $\varrho^{2}=y_{1}^{\prime 2}+y_{2}^{\prime 2}$ and $\mathbf{x}_{ \pm}$are $\delta$-offsets of its orthogonal projection $\stackrel{\nabla}{\mathbf{y}}$, which are rational PH curves as well. Clearly, $\mathbf{y}$ and $\underset{\nabla}{\mathbf{y}}$ are only translated versions of the same PH curve. Since $r^{\prime} \equiv 0$, (42) simplifies to

$$
\left(\sigma_{+} \kappa_{+}\right)^{2}\left(\sigma_{-} \kappa_{-}\right)^{2}=\varrho^{4} \varkappa^{4}
$$

yielding an interesting relation between a planar (rational) $\mathrm{PH}$ curve and its both-sided offsets.

We investigate this special case in more detail. Let $\varkappa$ be the (signed Euclidean) curvature $\varkappa=\left(y_{1}^{\prime} y_{2}^{\prime \prime}-y_{1}^{\prime \prime} y_{2}^{\prime}\right) / \varrho^{3}$ of $\stackrel{\nabla}{\mathbf{y}}$ (this can differ from the Minkowski curvature (17) of $\mathbf{y}$ by its sign only). Moreover, let $R=1 / \varkappa, R_{ \pm}=1 / \kappa_{ \pm}$be 


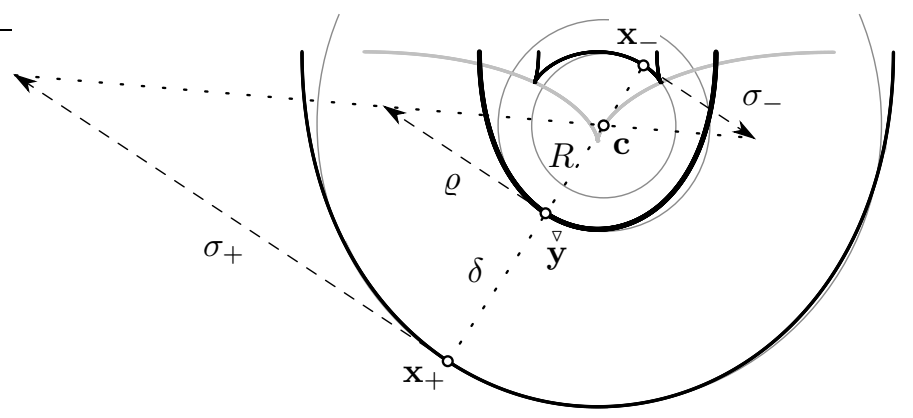

Figure 5: A PH curve $\stackrel{\nabla}{\mathbf{y}}$ with its $\delta$-offsets $\mathbf{x}_{ \pm}$, their speeds $\varrho, \sigma_{ \pm}$, and the corresponding concentric osculating circles (thin grey) with radii $R, R \pm \delta$ and with the center $\mathbf{c}$ lying on the common evolute (thick grey).

the signed (oriented) radii of curvature of ${ }^{\nabla}$ and $\mathbf{x}_{ \pm}$, respectively. It is well known (see e.g. Farouki and Neff (1990); Farouki (2008)) that offsets share a common evolute, i.e., the locus of all centers of curvature, see Fig. 5. Therefore, $R_{ \pm}=R \mp \delta$ and $\kappa_{ \pm}=\varkappa /(1 \mp \varkappa \delta)$. This in turn gives $1 \pm \kappa_{ \pm} \delta=\kappa_{ \pm} / \varkappa$. Comparing the last equation with (29) and (40) yields

$$
\sigma_{+}^{2} \kappa_{+}^{2}=\varrho^{2} \varkappa^{2}=\sigma_{-}^{2} \kappa_{-}^{2},
$$

which is a stronger result than (43).

\section{Conclusion}

In the present paper we introduced a novel approach for studying MPH curves. Since this approach overcomes the limitations of hodograph representation, it was successfully applied to rational MPH curves. Using the concept of associated planar PH and spatial MPH curves, we showed that any rational MPH curve in $\mathbb{R}^{2,1}$ can be obtained in terms of a rational PH curve in $\mathbb{R}^{2}$ and an extra rational function. We also studied relations between the Minkowski curvature of MPH curves and the curvatures of the associated planar PH curves. As a special case, our results can be applied to planar rational $\mathrm{PH}$ curves and their offsets.

The techniques presented in this paper might stimulate further research in the topic of Pythagorean hodograph curves and help to improve the understanding of these special classes of curves both in Euclidean plane and Minkowski space. In the literature there exist many efficient, yet separate techniques for various types of geometric interpolation designed either for polynomial/rational PH or MPH curves. Therefore, our approach can serve as a first step for formulating new interpolation algorithms based on the same unifying principle.

As we already mentioned in Remark 4, our study of associated PH and MPH curves in $\mathbb{R}^{2,1}$ is analogous to the characterization of MOS surfaces via PN surfaces in $\mathbb{R}^{3,1}$ by Peternell (2010). Nevertheless, the approach to MPH curves used in the present paper, based mainly on symbolic manipulations, led to new interesting results relating curvatures of involved PH and MPH curves (see Section 3.3). This fact might be helpful in formulating potential $\mathrm{G}^{2}$ interpolation algorithms. However, it still remains a challenge to discover whether similar relations hold for associated PN and MOS surfaces as well.

\section{Acknowledgments}

The work on this paper was supported by the Research Plan MSM 4977751301. A major part of the work was done during a visit of M. Lávička at the Centre of Mathematics for Applications, University of Oslo in May 2009.

We thank all referees for their comments, which helped us to improve the paper.

\section{References}

Choi, H.I., Han, Ch.Y., Moon, H.P., Roh, K.H., Wee, N.S., 1999. Medial axis transform and offset curves by Minkowski Pythagorean hodograph curves, Computer-Aided Design 31, 59-72. 
Choi, H.I., Lee, D.S., Moon, H.P., 2002. Clifford algebra, spin representation and rational parameterization of curves and surfaces, Advances in Computational Mathematics 17, 5-48.

Elber, G., Lee, I.-K., Kim, M.-S., 1998. Comparing Offset Curve Approximation Methods, IEEE Comp. Graphics and Appl. 17, 62-71.

Farouki, R.T., 2002. Pythagorean-hodograph curves, in: Hoschek, J. et al. (Eds.), Handbook of Computer Aided Geometric Design, Elsevier, pp. 405-427.

Farouki, R.T., 2008. Pythagorean-hodograph curves: Algebra and Geometry Inseparable, Springer, Berlin.

Farouki, R.T., al-Kandari, M., Sakkalis, T., 2002. Hermite interpolation by rotation-invariant spatial Pythagorean-hodograph curves, Advances in Computational Mathematics 17, 369-383.

Farouki, R.T., Han, Ch.Y., Manni, C., and Sestini, A., 2004. Characterization and construction of helical polynomial space curves, J. Comput. Appl. Math. 162, 365-392.

Farouki, R.T., Neff, C.A., 1990. Analytic properties of plane offset curves, Computer Aided Geometric Design 7, 83-99.

Farouki, R.T., Pottmann, H., 1994. Polynomial and Rational Pythagorean-Hodograph Curves Reconciled, Proceedings of the 6th IMA Conference on the Mathematics of Surfaces, 355-378 .

Farouki, R.T., Sakkalis, T., 1990. Pythagorean hodographs, IBM Journal of Research and Development 34, 736-752.

Farouki, R.T., Sakkalis, T., 1994. Pythagorean-hodograph space curves, Adv. Comput. Math. 2, 41-66.

Jüttler, B., Mäurer, C., 1999. Cubic Pythagorean Hodograph Spline Curves and Applications to Sweep Surface Modeling, Comp. Aided Design 31, $73-83$.

Kim, G.-I., Ahn M.-H., 2003. $C^{1}$ Hermite interpolation using MPH quartic, Computer Aided Geometric Design 20, 469-492.

Kosinka, J., Jüttler, B., 2006. Cubic Helices in Minkowski Space, Sitzungsber. Oesterr. Akad. Wiss. 215, Abt. II., 13-35.

Kosinka, J., Jüttler, B., 2006. $G^{1}$ Hermite Interpolation by Minkowski Pythagorean Hodograph Cubics, Comp. Aided Geom. Design 23, $401-418$.

Kosinka, J. and Jüttler, B., 2009. $C^{1}$ Hermite Interpolation by Pythagorean Hodograph Quintics in Minkowski space, Advances in Applied Mathematics 30, 123-140.

Kosinka, J., Šír, Z., 201x. $C^{2}$ Hermite Interpolation by Minkowski Pythagorean Hodograph Curves and Medial Axis Transform Approximation, Comp. Aided Geom. Design, in press, doi:10.1016/j.cagd.2010.04.005.

Krasauskas, R., Mäurer, C., 2000. Studying cyclides with Laguerre geometry, Computer Aided Design 17, 101-126.

Kubota, K.K., 1972. Pythagorean triples in unique factorization domains, American Mathematical Monthly 79, 503-505.

Maekawa, T., 1999. An overview of offset curves and surfaces, Computer Aided Design 31, 165-73.

Moon, H.P., 1999. Minkowski Pythagorean hodographs, Computer Aided Geometric Design 16, 739-753.

Pekerman, D., Elber, G., Kim, M.-S., 2008. Self-intersection detection and elimination in freeform curves and surfaces, Computer Aided Design 40, 150-159.

Peternell, M., 2010. Rational two-parameter families of spheres and rational offset surfaces, Journal of Symbolic Computation 45, 1-18.

Peternell, M., Pottmann, H., 1998. A Laguerre geometric approach to rational offsets, Computer Aided Geometric Design 15, $223-249$.

Pottmann H., 1995. Rational curves and surfaces with rational offsets, Computer Aided Geometric Design 12, 175-192.

Pottmann, H., Peternell, M., 1998. Applications of Laguerre geometry in CAGD, Computer Aided Geometric Design 15, 165-186.

Seong, J.-K., Elber, G., Kim, M.-S., 2006. Trimming local and global self-intersections in offset curves/surfaces using distance maps, Computer Aided Design 38, 183-93.

Šír, Z., Jüttler, B., 2005. Spatial Pythagorean Hodograph Quintics and the Approximation of Pipe Surfaces, in: Ralph R.M. et al. (Eds.), Lecture Notes on Computer Science 3604, Springer, Berlin, pp. 364-380.

Šír, Z., Jüttler, B., 2007. $C^{2}$ Hermite interpolation by spatial Pythagorean hodograph curves, Math. Comp. 76, 1373-1391.

Šír, Z., Kosinka, J., 2010. Low Degree Euclidean and Minkowski Pythagorean Hodograph Curves, in: M. Daehlen et al. (Eds.), Lecture Notes on Computer Science 5862, Springer, Berlin, pp. 394-418. 